Increased duration of aspirin use was also associated with progressively reduced risk of colorectal cancer, although significant benefit was not evident until more than 10 years' usage. The results for non-aspirin NSAIDs were comparable with those for aspirin. As well as the apparent benefit of aspirin and NSAIDs, increased dosages were associated with increased gastrointestinal bleeding.

The investigators note that further studies to address the risk-benefit profile of long-term aspirin or NSAID use in various risk groups, compared with other potential cancer prevention strategies, are warranted.

Rachel Murphy

Original article Chan AT et al. (2005) Long-term use of aspirin and nonsteroidal anti-inflammatory drugs and risk of colorectal cancer. JAMA 294: 914-923

\section{Liver transplantation or resection for hilar cholangiocarcinoma?}

Therapy for hilar cholangiocarcinoma (CCA) remains a challenge. Curative resection is often not an option owing to vascular encasement, hilar invasion, bilateral liver involvement and primary sclerosing cholangitis; however, to date, liver transplantation has achieved limited long-term survival in the majority of patients. Rea and colleagues have designed a protocol of neoadjuvant chemoradiotherapy and orthotopic liver transplantation for patients with unresectable hilar CCA, and compared survival and recurrence rates with patients treated with conventional resection.

Between January 1993 and August 2004, 38 patients received a liver transplant and 54 underwent resection; 26 of these surgeries were intended to be curative. For the liver transplant recipients, survival at 1,3 and 5 years following the procedure was $92 \%$, $82 \%$ and $82 \%$ respectively. In the 26 patients receiving potentially curative resection, survival was significantly lower: $82 \%, 48 \%$ and $21 \%$ at the 1, 3 and 5-year follow-up points. The incidence of tumor recurrence was also higher in the resection patients than the transplant recipients, and recurrence appeared sooner.

The authors conclude, despite the difficulty in comparing the two groups, that their liver transplantation protocol achieves higher survival with less recurrence than potentially curative resection in patients with node-negative, localized hilar CCA, and should be considered as an alternative treatment option in such patients.

Katy Cherry

Original article Rea DJ et al. (2005) Liver transplantation with neoadjuvant chemoradiation is more effective than resection for hilar cholangiocarcinoma. Ann Surg 242: 451-461

\section{Spinal cord compression due to metastasis: surgical resection is superior to radiotherapy}

A randomized multicenter trial has demonstrated that surgical resection is more effective than radiotherapy in patients undergoing surgery for spinal cord compression caused by metastatic cancer. Although standard treatment is radiotherapy and steroids, data from uncontrolled surgical studies encouraged Patchell et al. to investigate the effectiveness of surgical treatment, using ambulation as a primary endpoint.

The original intention to randomize 100 patients into two treatment groups-one receiving surgery within $24 \mathrm{~h}$ of randomization followed by radiotherapy, the other beginning radiotherapy alone within $24 \mathrm{~h}$. Both groups received steroids. Recruitment was halted, however, after interim analysis showed surgical treatment to be superior in terms of ambulation rates (84\% vs $57 \%, P=0.001$ ), duration of ambulatory ability, recovery of ambulatory ability, and use of corticosteroids and analgesics.

Eligible patients fulfilled strict criteria, including tissue-proven cancer diagnosis (not radiosensitive cancer, or cancer of CNS or spinal column origin), a single point of MRI-confirmed spinal-cord displacement, no unrelated neurological problems, and no previous spinal radiation preventing delivery of the study radiation dose. Also, patients could not have had total paraplegia for $>48 \mathrm{~h}$ prior to study entry. Although the authors concede that their conclusions cannot be extrapolated to patients not fulfilling these criteria, the study shows surgical treatment to be markedly superior to radiotherapy in this context.

Rebecca Doherty

Original article Patchell RA et al. (2005) Direct decompressive surgical resection in the treatment of spinal cord compression caused by metastatic cancer: a randomised trial. Lancet 366: 643-648 